\title{
Uma comparação entre a medição e a classificação da ruptura do manguito rotador no pré-operatório e no intraoperatório*
}

\section{A Comparison Between Preoperative and Intraoperative Measurement and Classification of the Size of Rotator Cuff Tears}

\author{
Karl Peter Gill1,20

\footnotetext{
te Fisioterapia, Northern Care Alliance NHS

Profissões da Saúde, Faculty of Health, Psychol Grenter,

3 Departamento de Ombro de Derby, University Hospitals Derby and
}

Marcus Bateman ${ }^{3(1)}$ Bruno Mazuquin ${ }^{2(1)}$ Chris Littlewood ${ }^{2}$

Endereço para correspondência Karl Peter Gill, PhD, Department of Health Professions, Manchester Metropolitan University, Manchester, M15 6GX, United Kingdom (e-mail: P.Gill@mmu.ac.uk).
Rev Bras Ortop

\author{
Resumo \\ Palavras-chave \\ - lesões do manguito \\ rotador \\ - ombro \\ - imagem por \\ ressonância \\ magnética \\ - ultrassonografia

Resumo
Palavras-chave
- lesões do manguito
rotador
- ombro
- imagem por
ressonância
magnética
- ultrassonografia

* Estudo realizado no Departamento de Profissões da Saúde, Faculty of Health, Psychology and Social Care, Manchester Metropolitan University, Manchester, Reino Unido. manguito rotador. recebido

18 de Maio de 2021

aceito

20 de Setembro de 2021
Objetivo Avaliar a concordância no tamanho de ruptura obtido por imagem préoperatória e por medição intraoperatória, e determinar a precisão da imagem préoperatória na classificação do tamanho da ruptura e na identificação de rupturas em cada tendão do manguito rotador.

Métodos Os dados de 44 pacientes recrutados para um ensaio controlado randomizado foram revisados retrospectivamente. O tamanho e a localização do manguito rotador foram confirmados por ultrassom ou ressonância magnética préoperatórios, e avaliados durante a cirurgia. Um teste $t$ e o gráfico de Bland e Altman foram usados para determinar a concordância entre as medições pré-operatória e intraoperatória. Sensibilidade, especificidade, valor preditivo positivo (VPP) e valor preditivo negativo (VPN) foram calculados para o tamanho do rompimento e o envolvimento do tendão do

Resultados Houve boa concordância para medidas de tamanho da ruptura (91\%) e classificação (89\%) pré-operatória e durante a cirurgia. Ao classificar o tamanho da ruptura, a sensibilidade e o VPP foram elevados para rupturas de tamanho médio
DOI https://doi.org/ 10.1055/s-0041-1741445. ISSN 0102-3616. (c) 2022. Sociedade Brasileira de Ortopedia e Traumatologia. All rights reserved.

This is an open access article published by Thieme under the terms of the Creative Commons Attribution-NonDerivative-NonCommercial-License, permitting copying and reproduction so long as the original work is given appropriate credit. Contents may not be used for commercial purposes, or adapted, remixed, transformed or built upon. (https://creativecommons.org/ licenses/by-nc-nd/4.0/)

Thieme Revinter Publicações Ltda., Rua do Matoso 170, Rio de Janeiro, RJ, CEP 20270-135, Brazil 


\section{Abstract}

\section{Keywords}

- rotator cuff tears

- shoulder

- magnetic resonance imaging

- ultrasonography
(100\%), e menor para rupturas grandes (75\%), o que indica que todas as rupturas de tamanho médio, mas nem todas as grandes, foram identificadas pré-operatoriamente. Para a identificação de rupturas, a sensibilidade pré-operatória e o VPP foram maiores para o supraespinal (84\%), com sensibilidade e VPP progressivamente menores para o infraespinal (57\%), o subescapular (17\%), e o redondo menor (0\%).

Conclusões Por meio da imagem pré-operatória, pode-se medir ou classificar com precisão o tamanho da ruptura. Quando há discordância, não está claro se o tamanho da ruptura é subestimado no exame ou superestimado durante a cirurgia. A alta sensibilidade demonstra que uma ruptura do supraespinal é geralmente detectada por escaneamento. As sensibilidades mais baixas para o infraespinal e o subescapular indicam que a identificação de rupturas nestes tendões é menos precisa.

Purpose To evaluate the agreement in tear size obtained through preoperative imaging and intraoperative measurement, and to determine the accuracy of preoperative imaging in the classification of tear size and identification of tears in each rotator cuff tendon.

Methods Data from 44 patients recruited to a randomized controlled trial were reviewed retrospectively. Size and location of the rotator cuff tears were confirmed by either ultrasound or magnetic resonance imaging scans preoperatively and evaluated during surgery. A t-test and Bland and Altman plot were used to determine the agreement between the preoperative and intraoperative measurements. Sensitivity, specificity, positive predictive value (PPV) and negative predictive value (NPV) were calculated for tear size and involvement of the rotator cuff tendon.

Results There was good agreement in terms of the measurements (91\%) and classification (89\%) of the tear size preoperatively and during surgery. When classifying tear size, the sensitivity and PPV were high for medium-sized tears (100\%) and lower for large tears (75\%), reflecting that all medium-sized tears but not all large tears were identified preoperatively. For the preoperative identification of the tears, the sensitivity and PPV were highest for the supraspinatus (84\%), with progressively lower sensitivities and PPV for the infraspinatus (57\%), subscapularis (17\%) and teres minor (0\%).

Conclusions Through preoperative imaging, the measurement or classification of the tear size can be accurately performed. Where there is disagreement, it is unclear whether the tear size is either underestimated on the scan or overestimated during surgery. The high sensitivity demonstrates that a supraspinatus tear is usually detected by scan. The lower sensitivities for the infraspinatus and subscapularis indicate that the identification of tears in these tendons is less accurate.

\section{Introdução}

As rupturas do manguito rotador são uma causa comum de dor no ombro, e são diagnosticadas por meio de uma combinação de histórico do paciente, exame clínico, e diagnóstico por imagem. A imagem, seja por ultrassom (US) ou ressonância magnética (RM), é usada para determinar o tamanho e a localização da ruptura. ${ }^{1}$ Também permite a classificação de rupturas como pequenas, médias, grandes ou maciças. ${ }^{2}$ Entender o tamanho e a localização de uma ruptura do manguito rotador é importante para o planejamento cirúrgico. Se uma ruptura for maior do que o esperado com base em achados de imagem, por exemplo, a cirurgia pode demorar mais, e outras técnicas adicionais podem ser necessárias. ${ }^{3}$
Tanto o US quanto a RM têm sensibilidades e especificidades comparáveis para os diagnósticos de rupturas de manguito rotador. Uma revisão sistemática da Cochrane ${ }^{4}$ relatou que não houve diferenças em termos de sensibilidade e especificidade entre a RM e o US para detectar rupturas de manguito rotador de espessura total ou parcial. $O$ teste de referência mais comum é a artroscopia diagnóstica, que permite uma avaliação mais precisa das características da ruptura e confirma a intervenção planejada. ${ }^{4}$

Dado que ambas as modalidades de imagem são utilizadas na prática clínica e os achados são importantes para fins de planejamento cirúrgico, o principal objetivo deste artigo foi avaliar a concordância no tamanho da ruptura entre a 
imagem pré-operatória (por US ou RM) e a medição intraoperatória coforme utilizada pragmaticamente na triagem dos pacientes para a inclusão em um ensaio clínico, e determinar a sensibilidade, a especificidade, o valor preditivo positivo (VPP) e o valor preditivo negativo (VPN) das imagens préoperatórias na classificação do tamanho da ruptura. Outro objetivo foi determinar a sensibilidade, a especificidade, o VPP e o VPN na identificação de rupturas em cada tendão do manguito rotador.

\section{Métodos}

Este é um estudo retrospectivo em que se usaram dados de um ensaio controlado, randomizado (ECR) e multicêntrico ${ }^{5}$ que investigou diferentes estratégias de reabilitação após a cirurgia de reparação do manguito rotador. Uma revisão ética favorável foi concedida pelo Comitê de Ética em Pesquisa do País de Gales 5 Bangor em 31 de julho de 2018 (18/WA/0242).

\section{Participantes}

Os pacientes para o ECR foram recrutados em 5 hospitais do National Health Service (NHS, o Serviço Nacional de Saúde do Reino Unido) entre novembro de 2018 e novembro de 2019 de acordo com os seguintes critérios: idade maior de 18 anos e diagnóstico de uma ruptura do manguito rotador de espessura total não traumática e sintomática e com cirurgia marcada.

Os pacientes $(n=44)$ incluídos neste estudo foram submetidos a reparação cirúrgica do manguito rotador e dispunham de medições pré-operatórias e intraoperatórias.

Oito cirurgiões trataram pelo menos um participante do $\mathrm{ECR}^{5}$

\section{Medição e Classificação de Rupturas}

O tamanho, a classificação e a localização da ruptura do manguito rotador foram determinados durante o trabalho de diagnóstico pré-cirúrgico de rotina por US ou RM de acordo com as vias locais e a preferência clínica. O US foi avaliado por um radiologista consultor ou um ultrassonografista musculoesquelético experiente. A RM foi medida em direção anteroposterior utilizando as vistas axial, coronal e sagital. Os dados foram posteriormente obtidos a partir de notas clínicas. A medição intraoperatória do tamanho e a classificação foram feitas levando-se em consideração os instrumentos cirúrgicos e realizadas pelo cirurgião em consonância com sua prática habitual. Como uma abordagem pragmática foi tomada, a padronização do procedimento, pré- ou intraoperatória, não foi aplicada. Os pacientes envolvidos neste estudo apresentaram rupturas de manguito rotador classificadas como médias $(1-3 \mathrm{~cm})$ ou grandes $(3-5 \mathrm{~cm}){ }^{2}$

\section{Análise de Dados}

Um teste- $t$ foi usado para determinar quaisquer diferenças entre as medidas pré-operatórias e intraoperatórias de tamanhos de ruptura (em centímetros).

Um gráfico de Bland e Altman determinou a concordância entre o tamanho relatado da ruptura no pré e no intraoperatório.

Sensibilidade, especificidade, VPP e VPN foram calculados para a classificação pré-operatória da ruptura (por US ou RM) e de cada tendão (ou seja, se uma ruptura foi identificada em um tendão específico) em relação aos achados intraoperatórios.

\section{Resultados}

Os dados pré- e intraoperatórios da ruptura estão relatados na - Tabela 1.

A diferença entre as medições pré- e intraoperatórias foi estatisticamente significativa com relação ao tamanho da ruptura $(0,3 \mathrm{~cm} ; p<0,01)$. Ao medir o tamanho da ruptura, $34 / 44(77 \%)$ dos casos apresentaram valores idênticos para as medidas pré- e intraoperatórias. Dos 10 pacientes (23\%) nos quais as medidas não eram idênticas, 7 (16)\% apresentaram

Tabela 1 Tamanho médio, classificação, e número de rupturas em cada tendão do manguito rotador identificados no pré e intraoperatório

\begin{tabular}{|l|l|l|l|}
\hline & Pré-operatório & Intraoperatório & $\begin{array}{l}\text { Correspondência } \\
\text { entre as duas medidas }\end{array}$ \\
\hline Tamanho da ruptura - média \pm desvio padrão & $2,4 \pm 1,2$ & $2,7 \pm 1,3$ & 34 \\
\hline Número de rupturas médias & 29 & 24 & 24 \\
\hline Número de rupturas grandes & 15 & 20 & 15 \\
\hline Total & 44 & 44 & 39 \\
\hline & $\begin{array}{l}\text { Número de rupturas } \\
\text { no pré-operatório }\end{array}$ & $\begin{array}{l}\text { Número de rupturas } \\
\text { no intraoperatório }\end{array}$ & $\begin{array}{l}\text { Número de rupturas } \\
\text { concordantes no pré- e } \\
\text { intraoperatório }\end{array}$ \\
\hline Supraespinal & 37 & 44 & 37 \\
\hline Infraespinal & 8 & 11 & 4 \\
\hline Subescapular & 5 & 6 & 1 \\
\hline Redondo menor & 0 & 1 \\
\hline
\end{tabular}




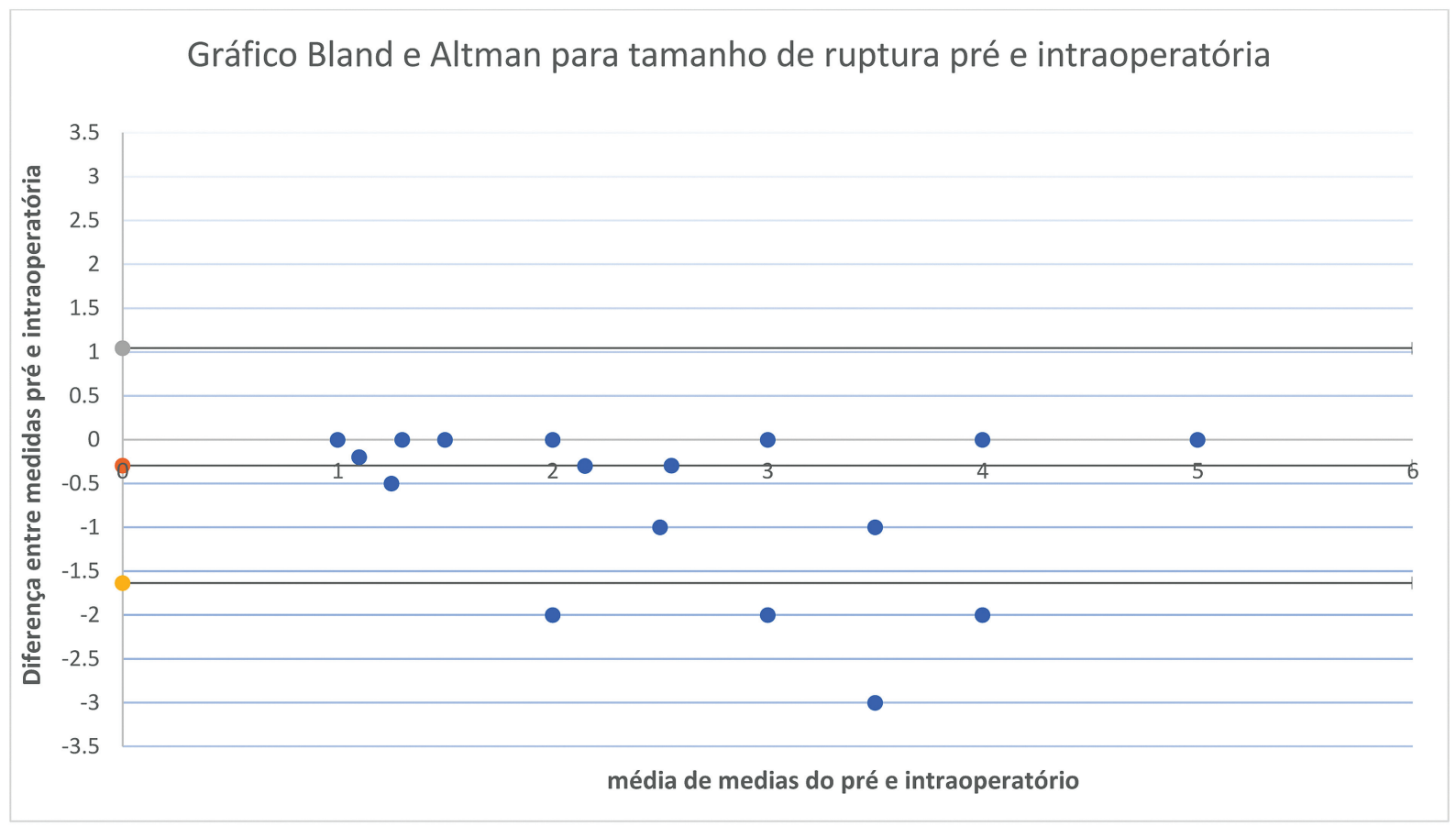

Limites superiores 1,043668061 1,634577152

Diferença média no tamanho da ruptura-0,295454545 Limites inferiores-

Fig. 1 Gráfico de Bland e Altman das rupturas no pré e intraoperatório. Limites superiores: 1,043668061; diferença média no tamanho do ruptura: $-0,295454545$; limites inferiores: $-1,634577152$.

diferença $\geq 1 \mathrm{~cm}$ entre as medidas pré- e intraoperatórias, e os $3(7 \%)$ restantes, uma diferença de $2 \mathrm{~cm}$.

Os limites de concordância foram de $1,36 \mathrm{~cm}$ no gráfico de Bland e Altman (-Figura 1), e, assim, apenas 4 (9\%) casos ficaram fora desses limites, resultando em uma concordância de $91 \%$ (40 casos) entre as medidas pré- e intraoperatórias.

Em relação à classificação do tamanho da ruptura (ou seja, média ou grande), houve concordância em 39/44 (89\%) casos. Os outros 5 (11\%) casos foram identificados como tendo rupturas grandes durante a cirurgia, mas que foram classificadas como de tamanho médio no exame pré-operatório.

A sensibilidade, a especificidade, o VPP e o VPN para a classificação da ruptura e cada tendão estão relatados na - Tabela 2. Os resultados para a classificação da ruptura indicam que todas as de tamanho médio, mas nem todas as grandes, foram identificadas no pré-operatório (cinco rupturas grandes na cirurgia foram classificadas como médias no pré-operatório). Uma sensibilidade de $84 \%$ (e VPP de 100\%) indica que, quando uma ruptura no tendão do supraespinal está presente, ela geralmente é identificada no exame. As sensibilidades de $57 \%$ para o infraespinal e de $17 \%$ para o subescapular indicam dificuldade crescente em identificar um rompimento desses tendões em um exame (baixo número de verdadeiros positivos, com um número relativamente alto de falsos negativos). Por outro lado, a alta especificidade e o VPN para o infraespinhal (89\%), o subescapular (89\%) e o redondo menor (100\%) indicam um número relativamente baixo de falsos positivos em relação ao grande número de verdadeiros negativos (com rupturas nesses músculos sendo menos comuns). Durante a cirurgia, o tendão do supraespinhal foi encontrado roto em todos os casos, mas, houve relativamente menos rupturas em outros tendões do manguito rotador: infraespinal em $25 \%$, subescapular em $13,6 \%$, e redondo menor em $2 \%$ dos casos.

Tabela 2 Sensibilidade, especificidade, e valores preditivos positivos e negativos para o tamanho da ruptura e para cada tendão do manguito rotador

\begin{tabular}{|l|l|l|l|l|}
\hline & Sensibilidade & Especificidade & Valor preditivo positivo & Valor preditivo negativo \\
\hline Rupturas médias & $100 \%$ & $75 \%$ & $83 \%$ & $100 \%$ \\
\hline Rupturas grandes & $75 \%$ & $100 \%$ & $100 \%$ & $83 \%$ \\
\hline Supraespinal & $84 \%$ & 0 & $100 \%$ & 0 \\
\hline Infraespinal & $57 \%$ & $89 \%$ & $50 \%$ & $92 \%$ \\
\hline Subescapular & $17 \%$ & $89 \%$ & $20 \%$ & $87 \%$ \\
\hline Redondo menor & 0 & $100 \%$ & 0 & $98 \%$ \\
\hline
\end{tabular}




\section{Discussão}

Neste estudo, a avaliação pré-operatória da integridade do manguito rotador envolveu US ou RM, que se mostraram comparáveis, com sensibilidades e especificidades semelhantes. ${ }^{4}$ Este estudo encontrou boa concordância entre a imagem pré-operatória e a avaliação intraoperatória em termos de medição e classificação do rompimento do manguito rotador. Em 5 dos 44 casos (11\%), o exame pré-operatório foi relatado como subestimando o tamanho das rupturas, e elas foram reclassificadas de médias a grandes quando avaliadas no intraoperatório. A razão para essa discordância não está clara, mas pode ser devida a uma subestimação ao visualizar os exames préoperatórios. Como as rupturas são em três dimensões, a orientação das imagens pode não ter revelado toda a extensão da ruptura, pois elas podem ter diferentes formas, como de U, de crescente, e de L. Em um estudo ${ }^{6}$ anterior, foram relatados diferentes graus de dificuldade na identificação de certas rupturas (especialmente em forma de L) ao se usar artrografia por RM em comparação com os achados artroscópicos. Por outro lado, a falta de uma abordagem padronizada para a medição intraoperatória pode ter contribuído para discordâncias na medição e classificação, uma vez que a ruptura pode ter sido medida de forma a relatar o maior valor, ou ter sido esgarçada ou distorcida para acomodar a ferramenta utilizada para medição. A ruptura também pode ter progredido em tamanho no período entre a tomografia pré-operatória e a cirurgia, o que é outra fonte potencial de discordância. ${ }^{7}$

Um segundo objetivo foi determinar a sensibilidade, a especificidade, o VPP e o VPN para a localização da ruptura do manguito rotador. Neste estudo, observou-se que o US préoperatório ou a RM em geral subnotificavam a frequência de rupturas nos tendões do manguito rotador (-Tabela 2 ). 0 escaneamento pré-operatório foi mais sensível na identificação de rupturas do supraespinhal, mas progressivamente menos sensível na identificação de rupturas do infraespinal, do subescapular, e do redondo menor. Uma pesquisa ${ }^{8}$ anterior que analisou a concordância entre US e RM encontrou um padrão semelhante, com maior concordância em relação ao supraespinhal e ao infraespinal, e com a avaliação do subescapular como a fonte de maior discordância. A dificuldade de identificar um local específico de ruptura do manguito rotador pode ser devida à natureza íntima do manguito rotador e à fusão dos tendões (em vez de serem estruturas distintas) à medida que se inserem no úmero e, como tal, a interpretação do US ou da RM pré-operatórios e a identificação do tendão durante a cirurgia podem estar sujeitas a erro.

\section{Pontos fortes e limitações}

Adotou-se uma abordagem pragmática, na qual o US ou a RM foram utilizados como parte do trabalho de diagnóstico de rotina antes da cirurgia. A medição intraoperatória do tamanho da ruptura foi uma estimativa em relação aos instrumentos cirúrgicos. Os dados foram extraídos de notas clínicas, e os processos de medição não foram padronizados. Desconhece-se a forma como a escolha da modalidade de imagem e o método de notificação contribuíram para qual- quer discordância. No entanto, apesar da natureza pragmática das medições deste estudo, a boa concordância entre exames pré-operatórios e os achados intraoperatórios para o tamanho da ruptura e os tendões envolvidos é reconfortante do ponto de vista do planejamento cirúrgico.

\section{Conclusões}

Imagens pré-operatórias, por US ou RM, podem ser usadas para medir ou classificar com precisão rupturas do manguito rotador (médias ou grandes). Quando há discordância, não está claro se as rupturas são subestimadas durante o escaneamento pré-operatório ou superestimadas durante a avaliação intraoperatória.

A alta sensibilidade demonstra que uma ruptura do supraespinhal é geralmente detectada pelo US ou pela RM pré-operatórios, mas a identificação de uma ruptura nos tendões infraespinal, subescapular ou redondo é mais problemática.

\section{Apoio Financeiro}

O ECR do qual se derivaram estes dados foi financiado pelo programa de Pesquisa para o Benefício do Paciente (PBPG-0816-20009) do National Institute for Health Research (NIHR). CL é bolsista de pós-doutorado do NIHR (PDF2018-11-ST2-005). As opiniões expressas são dos autores, e não necessariamente do NIHR ou do Departamento de Saúde e Assistência Social.

\section{Conflito de Interesses}

Os autores não têm conflito de interesses a declarar.

\section{Referências}

1 Spencer EE Jr, Dunn WR, Wright RW, et al Shoulder Multicenter Orthopaedic Outcomes Network. Interobserver agreement in the classification of rotator cuff tears using magnetic resonance imaging. Am J Sports Med 2008;36(01):99-103

2 DeOrio JK, Cofield RH. Results of a second attempt at surgical repair of a failed initial rotator-cuff repair. J Bone Joint Surg Am 1984;66(04):563-567

3 Chalmers PN, Tashjian RZ. Patch Augmentation in Rotator Cuff Repair. Curr Rev Musculoskelet Med 2020;13(05):561-571

4 Lenza M, Buchbinder R, Takwoingi Y, Johnston RV, Hanchard NC, Faloppa F. Magnetic resonance imaging, magnetic resonance arthrography and ultrasonography for assessing rotator cuff tears in people with shoulder pain for whom surgery is being considered. Cochrane Database Syst Rev 2013;2013(09):CD009020

5 Littlewood C, Bateman M, Butler-Walley S, et al. Rehabilitation following rotator cuff repair: A multi-centre pilot \& feasibility randomised controlled trial (RaCeR). [published online ahead of print, 2020 Dec 11]Clin Rehabil 2021;35(06):829-839

6 Lee YH, Kim AH, Suh JS. Magnetic resonance visualization of surgical classification of rotator cuff tear: comparison with threedimensional shoulder magnetic resonance arthrography at 3.0 $\mathrm{T}$. Clin Imaging 2014;38(06):858-863

7 Yamamoto N, Mineta M, Kawakami J, Sano H, Itoi E. Risk Factors for Tear Progression in Symptomatic Rotator Cuff Tears: A Prospective Study of 174 Shoulders. Am J Sports Med 2017;45(11): 2524-2531

8 Fischer CA, Weber MA, Neubecker C, Bruckner T, Tanner M, Zeifang F. Ultrasound vs. MRI in the assessment of rotator cuff structure prior to shoulder arthroplasty. J Orthop 2015;12(01):23-30 